\title{
Clinical and Epidemiological Metabonomics
}

\author{
Mika Ala-Korpela, ${ }^{1,2,3,4}$ Veikko Salomaa, ${ }^{5}$ and Olav M. Kvalheim ${ }^{6,7}$ \\ ${ }^{1}$ College of Chemistry and Chemical Engineering, Central South University, Changsha 410083, Hunan Province, China \\ ${ }^{2}$ Computational Medicine Research Group, Institute of Clinical Medicine, Faculty of Medicine, \\ University of Oulu and Biocenter Oulu, P.O. Box 5000, 90014 University of Oulu, Oulu, Finland \\ ${ }^{3}$ NMR Metabonomics Laboratory, Department of Biosciences, University of Eastern Finland, 70211 Kuopio, Finland \\ ${ }^{4}$ Department of Internal Medicine and Biocenter Oulu, Clinical Research Center, \\ University of Oulu, 90014 University of Oulu, Oulu, Finland \\ ${ }^{5}$ Chronic Disease Epidemiology and Prevention Unit, Department of Chronic Disease Prevention, \\ The National Institute for Health and Welfare, 00271 Helsinki, Finland \\ ${ }^{6}$ Department of Chemistry, University of Bergen, 5007 Bergen, Norway \\ ${ }^{7}$ Faculty of Health Studies, Sogn og Fjordane University College, 6800 Førde, Norway \\ Correspondence should be addressed to Mika Ala-Korpela, mika.ala-korpela@computationalmedicine.fi
}

Received 20 November 2010; Accepted 20 November 2010

Copyright ( $) 2011$ Mika Ala-Korpela et al. This is an open access article distributed under the Creative Commons Attribution License, which permits unrestricted use, distribution, and reproduction in any medium, provided the original work is properly cited.

Metabonomics is an omics approach to identify and monitor metabolic characteristics, changes, and phenotypes with respect to various synergetic factors such as environment, life style, diet, and potential pathophysiological processes. Recently, metabonomics has also opened up a possibility for functional genomics in large genome-wide studies. Mass spectrometry (MS) and proton nuclear magnetic resonance (NMR) spectroscopy are the two key experimental technologies in the field. Recent advancements are numerous, and methodologies currently exist that allow for automated high-throughput experimentation in a very costeffective manner. Technical developments have evoked an increasing number of metabonomics applications in clinical and epidemiological disciplines to understand the complex molecular foundations of various diseases. Techniques with the aim of assessing large numbers of metabolites that are substrates, intermediates, or products in various metabolic pathways are particularly relevant in the risk assessment of metabolic conditions like diabetes and vascular diseases. It has also been envisioned that metabonomics approaches may overtake standard analytical measurements of individual metabolites and eventually lead to holistic multimetabolic risk phenotyping in the early detection of high-risk individuals for various metabolic diseases. Thus, metabonomics offers potential means to move away from single biomarkers and thresholds in clinical medicine. And, furthermore, even though the current medical biomarkers and thresholds are necessary benchmarks for clinical practice, it will be crucial to let the future diagnostics to follow from new science. Good predictions are necessary for effective prevention; an important step towards this is expected to happen when complex traits will not anymore be limited by clinical diagnoses and definitions but will be handled as multivariate continuous dimensions. Consequently, clinical and epidemiological metabonomics is likely to be one of the key new omics areas to assist translational research in the near future.

Here, the Journal of Biomedicine and Biotechnology presents this special issue, 2011. Although only representing a small fraction of the contribution of metabonomics to various biomedical disciplines, the papers presented here show the increasing potential of metabonomics approaches to complement clinical and epidemiological research.

To start the special issue, we have five contributions that review several aspects of metabonomics research. M. G. Barderas et al. discuss the concepts of metabolic profiling, fingerprinting, and footprinting focusing on the area of cardiovascular diseases. E. Silvestri and coworkers discuss the timely and relevant issue of integrative analyses of related 
omics data sets in order to furnish new insights not accessible through one-dimensional data. Further, I. Kouskoumvekaki and G. Panagiotou give an extensive up-to-date overview on the database resources on metabolic pathways and metabolomes and also summarize the statistical tools often used for the analysis of metabonomics data. Y. Kim and S. Milner deal with the challenges related to bioactive food components in cancer research and prevention. Moreover, M. Sofia and coauthors review the potential use of exhaled breath condensate as a suitable matrix for NMR-based metabonomics of airway diseases.

This special issue contains two methodology reports. G. Kastenmüller and coworkers have developed a freely accessible methodology to facilitate automated and standardized analysis for quantitative metabolic data, covering the steps from data acquisition to biological interpretation. M. C. Martínez-Bisbal and colleagues, using magnetic resonance microscopy, present a critical and valuable interpretation on the high-resolution magic angle spinning data of human tumor tissues.

The six original research papers in this special issue start with the paper by J. Willmann et al. who demonstrate the advantage of combining high-pressure liquid chromatography, MS, and NMR spectroscopy in analyzing three different types of extracts of a common membrane component phosphatidylcholine. Then, D. F. Brougham et al. focus on the use of artificial neural networks in classifying proton NMR spectroscopic data recorded on whole-cell culture samples of four different lung carcinoma cell lines, displaying different drug-resistance patterns. In a mouse study, D. Otter et al. apply MS-based nontargeted urinary metabolite profiling to identify biomarkers of colon inflammation. In another mouse study, J. Zhou et al. provide evidence that NMR metabonomics may be sensitive enough to detect small differences in the composition of maternal plasma and thus be helpful for identifying biomarkers of birth defects. Additionally, in low-birth weight and high-birth weight piglets P. M. Nissen and coworkers combine NMR and MS to reveal a relationship between birth weight and plasma inositols, suggesting their potential role in fetal programming of type 2 diabetes. L. Guo et al. end this special issue in providing the latest epidemiologic information on the etiology distribution of neurodevelopmental disabilities in Chinese, and by demonstrating, on the basis of 8-year clinical experience, that MS-based pediatric metabonomics is clinically helpful.

Overall, we hope that this special issue hints at the rising potential of metabonomics approaches in clinical and epidemiological research and translational medicine. 

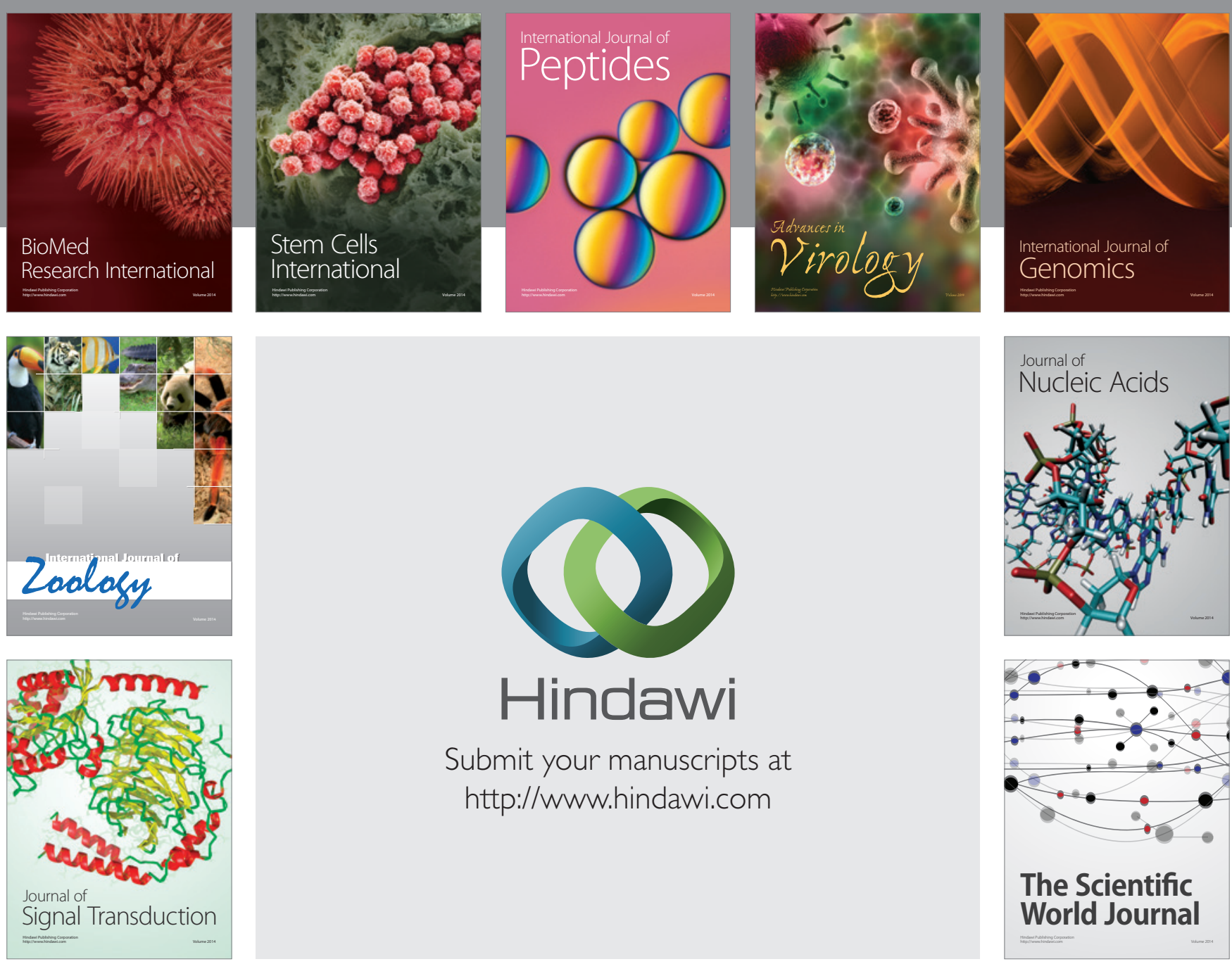

Submit your manuscripts at

http://www.hindawi.com
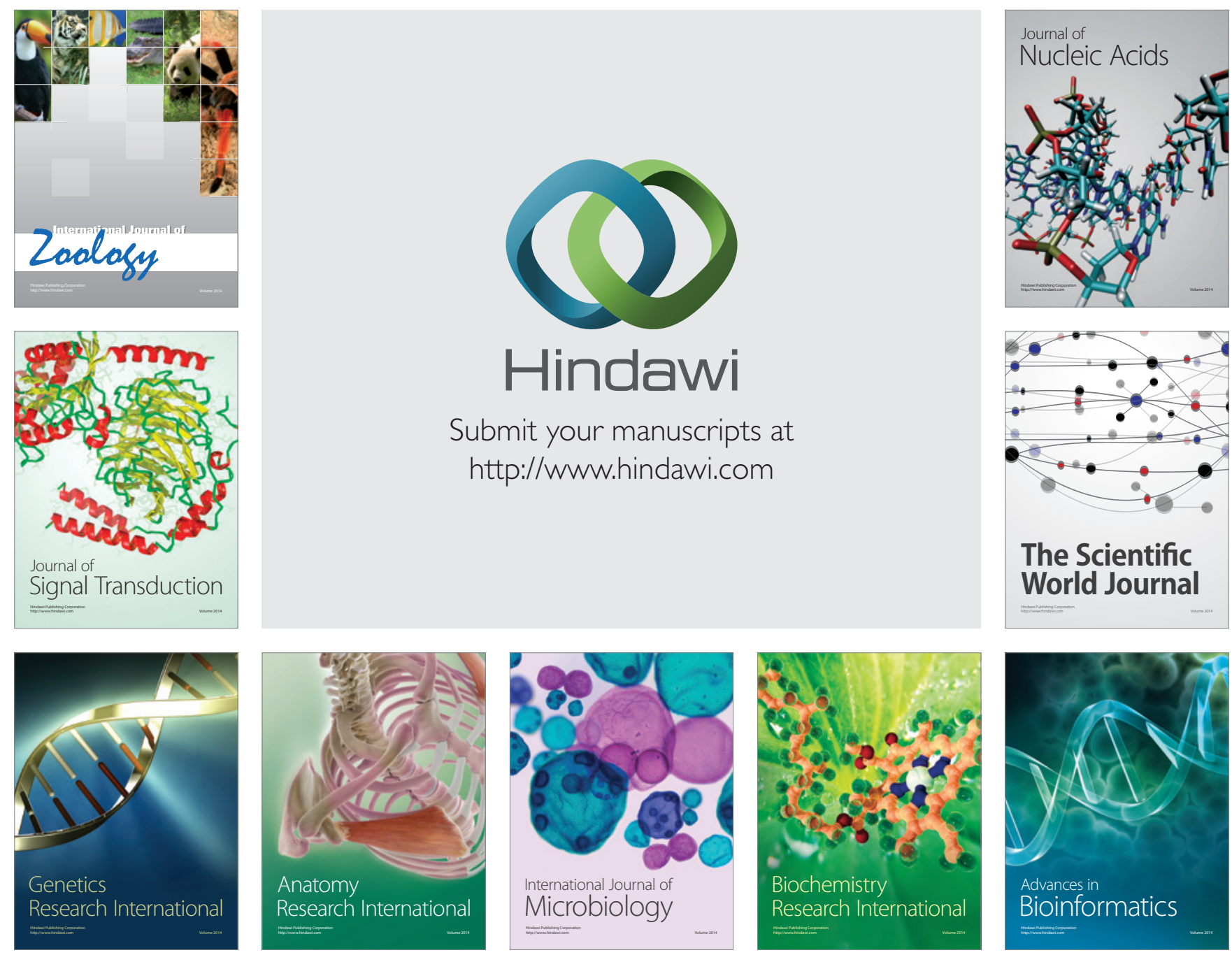

The Scientific World Journal
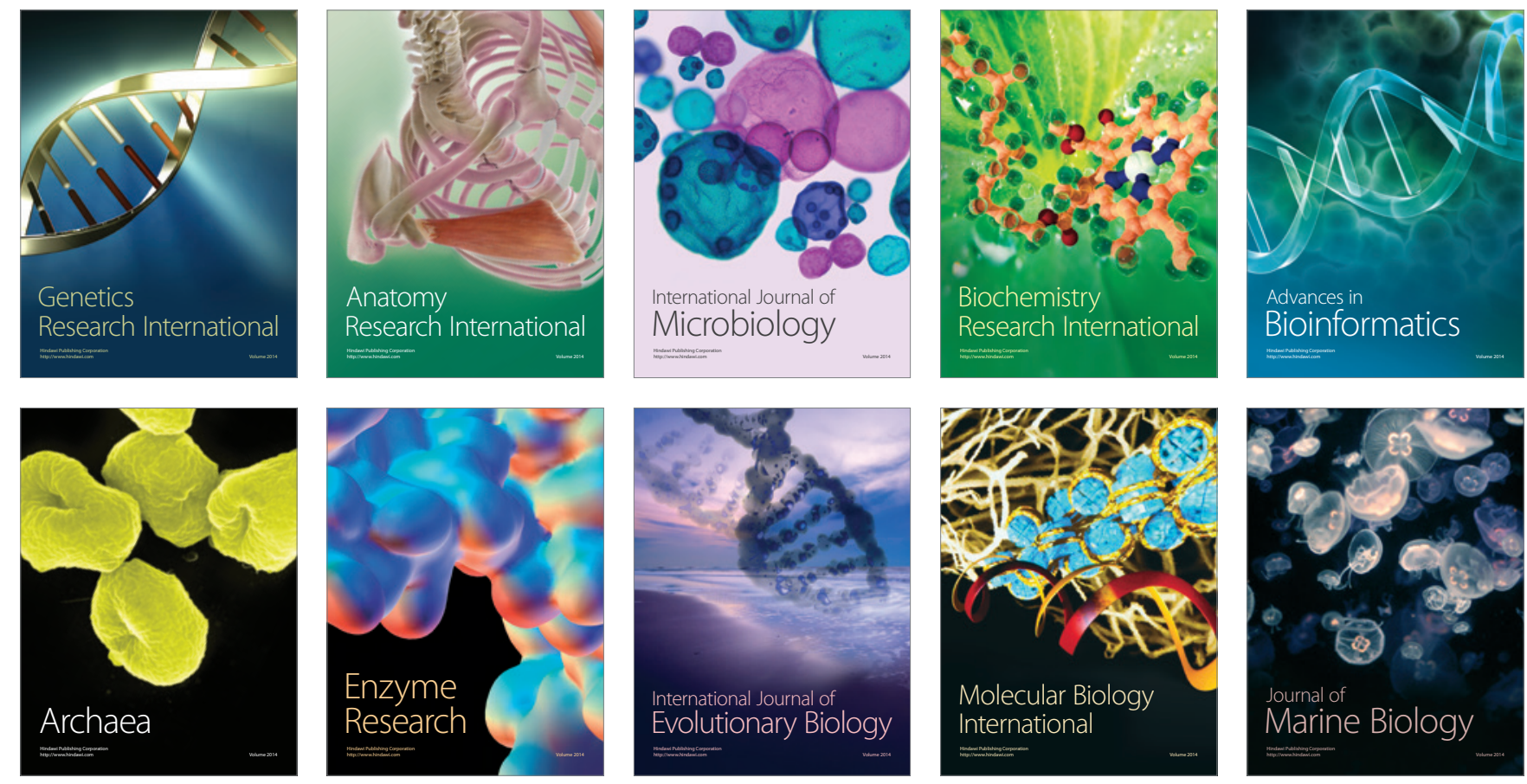